\title{
GMR
}

\section{Molecular authentication of Gynostemma pentaphyllum through development and application of random amplification polymorphic DNA sequence-characterized amplified region marker}

\author{
J. Zhou ${ }^{1,2}$, Y.S. Wu' ${ }^{1}$, R.Q. Zhao', J.F. Jiang' ${ }^{1}$, Y. Luo', C.T. Ma1 and J.Y. Qian ${ }^{1}$ \\ ${ }^{1}$ Department of Biochemistry and Molecular Biology, \\ Key Laboratory of Biological Molecular Medicine Research, \\ Guangxi Medical University, Nanning, Guangxi, China \\ ${ }^{2}$ College of Life Science and Technology, Center for Human Genome Research, \\ Huazhong University of Science and Technology, Wuhan, China \\ Corresponding author: Y.S. Wu \\ E-mail: wuyaosheng03@sina.com
}

Genet. Mol. Res. 14 (4): 16204-16214 (2015)

Received August 22, 2015

Accepted October 1, 2015

Published December 8, 2015

DOI http://dx.doi.org/10.4238/2015.December.8.10

ABSTRACT. Due to the morphological similarities of aerial parts, it is difficult to distinguish Gynostemma pentaphyllum from Cayratia japonica, which is usually an adulterant of the former. To develop a reliable method for the identification and authentication of G. pentaphyllum, a combination of random amplification polymorphic DNA (RAPD) technique with sequencecharacterized amplified region (SCAR) markers was studied. Twenty-five samples of $G$. pentaphyllum and two samples of $C$. japonica were collected from different regions in Guangxi or bought from different provinces in China. Through the RAPD analysis, significant genetic polymorphism was observed among the intraspecies samples of $G$. pentaphyllum. Furthermore, a specific marker, J-750, was obtained for authentication. 
Therefore, the SCAR marker for G. pentaphyllum (359 bp) was developed from the RAPD amplicon. With PCR amplification using the SCAR primers, a specific band of 359 bp was distinctly visible for all tested samples of $G$. pentaphyllum, but was absent in the samples of $C$. japonica. Furthermore, the results revealed that the SCAR marker was useful for the identification and authentication of $G$. pentaphyllum irrespective of whether samples were fresh, dry, or of commercial origin. The SCAR marker obtained in this study successfully authenticated G. pentaphyllum through an integrated PCR system containing SCAR and control primer combinations of two pairs. In addition, it was also used for simultaneous discrimination of $G$. pentaphyllum from $C$. japonica.

Key words: Sequence-characterized amplified region; Gynostemma pentaphyllum; Random amplification polymorphic DNA; Molecular authentication

\section{INTRODUCTION}

Gynostemma pentaphyllum (Thunb.) Makino (Jiaogulan) belongs to Gynostemma BL. of the family Cucurbitaceae. It is a widely distributed and abundant species in China and Southeast Asia (Liao et al., 2011) and has a complex genetic background (Jiang et al., 2009b; Liao et al., 2011). Many studies have shown that $G$. pentaphyllum extracts possess various biological properties, such as immunomodulatory (Im et al., 2012), anticancer (Liu et al., 2014; Deng and Yang, 2014; Tsui et al., 2014), antifatigue (Shan and Shi, 2014), and neuroprotective effects (Schild et al., 2009; Choi et al., 2010), and they are also involved in the regulation of lipid metabolism (Wang et al., 2013) and hypoglycemia (Gao et al., 2014; Park et al., 2014). Therefore, G. pentaphyllum has been extensively used in traditional Chinese medicine and in diverse industries such as healthcare, food, and cosmetics. However, the quality and safety of medications containing Jiaogulan are influenced by the presence of chaotic adulterants in the medicinal herb markets. In China, some medicinal plants have several synonymous names in different regions, or one name may represent different plants in disparate areas. For example, Cayratia japonica of the family Vitaceae is known as "Jiaogulan" in Shandong Province and in the north of Jiangsu Province (Ding 1998). Moreover, due to the morphological similarity of aerial parts, it is difficult to identify G. pentaphyllum and discriminate it from $C$. japonica, particularly in the case of dry medicinal materials. The morphologic characteristics of both $G$. pentaphyllum and $C$. japonica are similar, and they both possess leaves with pedate foliolates (usually 5-7), herbaceous, climbing, or prostrate stems, and are often found along brooks, in valleys, forests, thickets, and on roadsides on mountain slopes (Chen, 2007; Lu, 2011).

Authentication of genuine species has become a key issue for the standardization of herbal preparations in traditional medicine, because most of these medications are produced from herbs that are collected from wild habitats or are cultivated on farms. Although some methods have been developed to distinguish G. pentaphyllum from C. japonica based on morphotypes, microcharacters, or physical and chemical reactions, these are dependent on taxonomy experts. Currently, there are few reports about the authentication of G. pentaphyllum at the DNA molecular level. Therefore, the correct genotypic identification of plant material is essential in order to protect public health and for industrial production. 
Limitations of chemical and morphological approaches regarding the authentication of medicinal plants have resulted in the development of newer quality control techniques. Genetic polymorphisms in medicinal plants have been widely studied, and have helped to differentiate herbal medicines at the genomic level. Various molecular markers have been explored for use in the authentication of botanical materials such as ginseng (Wang et al., 2011b), atractylodes (Mizukami et al., 2000), and such techniques can provide more objective and reliable tools for the categorization and authentication of plant materials. Conventionally, randomly amplified polymorphic DNA (RAPD), based on polymerase chain reaction (PCR) amplification, has been used to estimate genetic diversity in wild plant populations or cultivars. This method can also be used to effectively authenticate various herbal medicines (Yang et al., 2013; Xin et al., 2014; Mei et al., 2014). However, the repeatability of results obtained by RAPD is dependent on the quality of DNA extracted from the botanical samples. Unfortunately, it is always difficult to obtain high-quality DNA from dry and processed herb materials. Therefore, a technique known as sequence-characterized amplified region (SCAR) has been developed based on RAPD (Bashir et al., 2014), which is specific, sensitive, and reproducible. SCAR markers have been explored and used in many medicinal plants, including Bulbus fritillariae (Xin et al., 2014), Pistacia chinensis (Sun et al., 2014), Acorus gramineus (Ryuk et al., 2014), Rosa indica (Bashir et al., 2014), Miscanthus sacchariflorus (Kim et al., 2012), and Eucommia ulmoides (Wang et al., 2011a). However, there are no suitable SCARs for G. pentaphyllum.

The objective of this study was to develop a method to distinguish G. pentaphyllum and $C$. japonica using DNA molecular markers. RAPD analysis of DNA from these two plants was completed by screening 20 decamer oligonucleotide primers. In light of the RAPD analysis results, a reliable SCAR marker was developed to enable the identification of these two species. Additionally, a PCR integrated system was developed to allow the simultaneous detection of these two herbal species through the conversion of RAPD to SCAR markers.

\section{MATERIAL AND METHODS}

\section{Plant material}

Twenty-five samples of $G$. pentaphyllum and two samples of $C$. japonica were used in this study. All plant materials were collected from different regions in Guangxi or bought from different provinces in China (Table 1). The botanical species were identified by Professor Kaijia He, Guangxi Institute of Chinese Medicine and Zhengzhu Ling in Guangxi Medicine Botanical Garden.

\section{Isolation of DNA}

Genomic DNA was extracted from fresh and dried samples using the cetyltriethylammonium bromide (CTAB) protocol with minor modifications, as previously described (Jiang et al., 2009a). DNA concentration and purity were determined by spectrophotometry (Smartspec ${ }^{\mathrm{TM}}$ plus, Bio-Rad, USA), and electrophoresis on a $1.5 \%$ agarose gel with known standards. Gels were stained using ethidium bromide and visualized under ultraviolet (UV) light. The final DNA concentration of each sample was diluted to approximately $40 \mathrm{ng} / \mu \mathrm{L}$ for PCR amplification.

\section{PCR amplification}

Twenty random decamer primers were used for RAPD amplification (Table 2), which were 
of high homology and aligned with the genome of Arabidopsis thaliana. The PCR reaction mixture contained $40 \mathrm{ng}$ template DNA in a $25-\mu \mathrm{L}$ reaction volume with $2.5 \mu \mathrm{L} 10 \mathrm{X}$ reaction buffer, $2 \mathrm{mmol} / \mathrm{L}$ $\mathrm{MgCl}_{2}, 100 \mu \mathrm{mol} / \mathrm{L}$ dNTPs, I U ExTaq DNA polymerase (Dalian TaKaRa Co. Ltd.), and $10 \mathrm{pmol}$ primer. Amplification was performed in a DNA thermal cycler (TGradient PCR, Biometra, Germany) using the following parameters: $94^{\circ} \mathrm{C}$ for $3 \mathrm{~min} ; 40$ cycles at $94^{\circ} \mathrm{C}$ for $50 \mathrm{~s}, 37^{\circ} \mathrm{C}$ for $50 \mathrm{~s}$, and $72^{\circ} \mathrm{C}$ for $2 \mathrm{~min}$, and a final extension at $72^{\circ} \mathrm{C}$ for $10 \mathrm{~min}$. PCR products were subjected to agarose gel $(1.5 \%[\mathrm{w} / \mathrm{v}])$ electrophoresis in $1 \mathrm{X}$ Tris acetate buffer, along with DL2000 DNA ladders (Beijing Sunbiotech Co. Ltd.) as size markers. Gels were stained using ethidium bromide and visualized under UV light.

Table 1. Information of the 27 samples (25 Gynostemma pontaphyllum, 2 Cayratia japonica) used in this study.

\begin{tabular}{|c|c|c|c|}
\hline No. & Species of herbal plant & Source (county, province, country) & Notes \\
\hline J & G. pentaphyllum & Nanning, Guangxi, China & Fresh material \\
\hline J2 & G. pentaphyllum & Lingyun, Guangxi, China & Fresh material \\
\hline J3 & G. pentaphyllum & Nanning, Guangxi, China & Fresh material \\
\hline $\mathrm{J} 4$ & G. pentaphyllum & Jingxiu, Guangxi, China & Fresh material \\
\hline J5 & G. pentaphyllum & Jingxiu, Guangxi, China & Fresh material \\
\hline J6 & G. pentaphyllum & Nanning, Guangxi, China & Fresh material \\
\hline J7 & G. pentaphyllum & Damingshan, Guangxi, China & Fresh material \\
\hline J8 & G. pentaphyllum & Jingxiu, Guangxi, China & Fresh material \\
\hline JG1 & G. pentaphyllum & Nanning, Guangxi, China & Dry material \\
\hline JG2 & G. pentaphyllum & Jinxiu, Guangxi, China & Commercial material \\
\hline JG3 & G. pentaphyllum & Nanning, Guangxi, China & Dry material \\
\hline JG4 & G. pentaphyllum & Jinxiu, Guangxi, China & Dry material \\
\hline JG5 & G. pentaphyllum & Jinxiu, Guangxi, China & Dry material \\
\hline JG6 & G. pentaphyllum & Nanning, Guangxi, China & Dry material \\
\hline JG7 & G. pentaphyllum & Damingshan, Guangxi, China & Dry material \\
\hline JG8 & G. pentaphyllum & Jinxiu, Guangxi, China & Dry material \\
\hline JG9 & G. pentaphyllum & Shanxi, China & Jiaogulan tea \\
\hline JG10 & G. pentaphyllum & Guizhou, China & Commercial material \\
\hline JG11 & G. pentaphyllum & Hunan, China & Commercial material \\
\hline JG12 & G. pentaphyllum & Changde, Hunan, China & Jiaogulan tea \\
\hline JG13 & G. pentaphyllum & Nanning, Guangxi, China & Dry material \\
\hline JG14 & G. pentaphyllum & Guilin, Guangxi, China & Commercial material \\
\hline JG15 & G. pentaphyllum & Lipu, Guangxi, China & Commercial material \\
\hline JG16 & G. pentaphyllum & Fusui, Guangxi, China & Commercial material \\
\hline JG17 & G. pentaphyllum & Jingxiu, Guangxi, China & Commercial material \\
\hline W1 & C. japonica & Lingyun, Guangxi, China & Fresh material \\
\hline W2 & C. japonica & Lingyun, Guangxi, China & Dry material \\
\hline
\end{tabular}

\section{Table 2. Sequences of random decamer primers used in this study.}

\begin{tabular}{clcl}
\hline No. & Primer sequence & No. & Primer sequence \\
\hline 1 & CTGCTGGGAC & 11 & GTTGCCAGCC \\
2 & GTAGACCCGT & 12 & ACTTCGCCAC \\
3 & CCTTGACGCA & 13 & TTCCGCCACC \\
4 & TCCGCTCTGG & 14 & TGGGGAGTG \\
5 & AGGGAACGAG & 15 & ACAGGTGCTG \\
6 & GGACCCTTAC & 16 & TGGCAAGGCA \\
7 & TTCCGAACCC & 17 & CCAAGAGGCT \\
8 & TGAGCGGACA & 18 & GAGCACTGCT \\
9 & CATCCGTGCT & 19 & TGGGCTCTGG \\
10 & CTCACCGTCC* & 20 & GTGTGCAGTG \\
\hline
\end{tabular}

*Amplified J-750 for Gynostemma pentaphyllum, SCAR marker. 


\section{Screening strategy and identification of specific RAPD amplicon}

A series of reproducible fragments, which migrated at the same rate, were amplified by primer 10 from 25 samples of G. pentaphyllum, including eight fresh and 17 dried materials. Bands of $750 \mathrm{bp}$ were generated from all G. pentaphyllum samples but were absent in samples from $C$. japonica species. Specific fragments of the RAPD amplicon from eight fresh $G$. pentaphyllum samples were selected and named J-750 for further cloning and sequencing.

\section{Cloning of RAPD amplicon}

The J-750 fragment amplified by primer 10, which is a putative marker, was excised from a $1.5 \%$ agarose gel, purified using a Gel Extraction Kit (Biospin, Hangzhou Bioer Technology Co. Ltd.), inserted into the pMD18-T vector (Dalian TaKaRa Co. Ltd.), and the recombinant vector was transformed into Escherichia coli JM-109 (MBI). Plasmid DNA was extracted using a Plasmid DNA Extraction Kit (Biospin, Hangzhou Bioer Technology Co. Ltd.). Positive clones were confirmed through profile analysis of double restriction digestion with BamHI and HindIII, and then sequenced by Beijing Sunbio Biotech Co. Ltd.

\section{Development of the SCAR marker integrated amplification system}

Based on the sequenced RAPD amplicon, a pair of SCAR oligonucleotide primers (G1 and G2) was designed along the hyperconservative region, which could amplify approximately $359 \mathrm{bp}$ of the genomic DNA of G. pentaphyllum. Two pairs of control primers were obtained according to the 18S rDNA sequences of Cucurbitaceae or Vitaceae, respectively, and were named J1-J2 and W1-W2. J1-J2 could amplify a 293-bp fragment within the 18S rDNA region of G. pentaphyllum; however, W1-W2 amplified only a 177-bp fragment in C. japonica. All primers (Table 3) were synthesized by Beijing Sunbio Tech Co. Ltd. The three pairs of primers were used for PCR amplifications of genomic DNA from all materials. Speciesspecific amplification was performed using a PCR integrated system with a SCAR primer pair and a control primer pair. Reactions were performed in a $25-\mu \mathrm{L}$ mixture containing two pairs of primers (10 pmol of each primer), $80 \mathrm{ng}$ DNA template, and the same buffer as previously described. Thermal cycling conditions for amplification were $94^{\circ} \mathrm{C}$ for $3 \mathrm{~min} ; 10$ cycles at $94^{\circ} \mathrm{C}$ for $30 \mathrm{~s}, 69^{\circ} \mathrm{C}$ for $30 \mathrm{~s}$, and $72^{\circ} \mathrm{C}$ for $30 \mathrm{~s}$. The annealing temperature was reduced by $1^{\circ} \mathrm{C}$ at every cycle until the "touchdown" annealing temperature reached $59^{\circ} \mathrm{C}$; then 20 cycles were performed at $94^{\circ} \mathrm{C}$ for $30 \mathrm{~s}, 59^{\circ} \mathrm{C}$ for $30 \mathrm{~s}$, and $72^{\circ} \mathrm{C}$ for $30 \mathrm{~s}$, with a final extension at $72^{\circ} \mathrm{C}$ for $10 \mathrm{~min}$.

\begin{tabular}{lll}
\multicolumn{2}{c}{ Table 3. Primer sequences for PCR integrated systems 1 and 2.} & Amplification region \\
\hline Primer & Primer sequence & SCAR of G. pentaphyllum \\
\hline G1 & ATAAGATGAACAGGCCATAAG & 18S rDNA of Cucurbitaceae \\
G2 & GTAAACTGACCCGAACACTC & 18S rDNA of Vitaceae \\
J1 & AGACTGTGAACTGCGAATGG & \\
W1 & CTCCGTCACCCGTCACC & \\
W2 & CGCGTGGGATCTGCCGACA \\
\hline
\end{tabular}




\section{Analysis of sequence data}

Alignment analysis of sequenced J-750 was performed using Vector NTI and Clustal W. Homology screening was performed using the BLAST algorithm within the GenBank database at http://www.ncbi.nlm.nih.gov/BLAST/ of National Center for Biotechnology Information, using the program BLASTn.

\section{RESULTS}

\section{Analysis and identification of RAPD marker for G. pentaphyllum}

High-molecular weight genomic DNA was extracted from various sources of fresh and dry materials of $G$. pentaphyllum and $C$. japonica (Table 1). Some degraded DNA fragments were observed in the DNA extracted from dry samples. Species-specific genomic sequences were screened using the RAPD method. Of the 20 random decamer primers selected, PCR with 19 primers separately produced a distinct and reproducible amplification profile for all samples of $G$. pentaphyllum. Among the 19 primers, primer 10 consistently amplified an intense band of approximately 750 bp only in $G$. pentaphyllum samples, but this band was absent in the $C$. japonica samples (Figure 1A-C). This band, named J-750, was selected as a putative-specific RAPD marker of G. pentaphyllum.
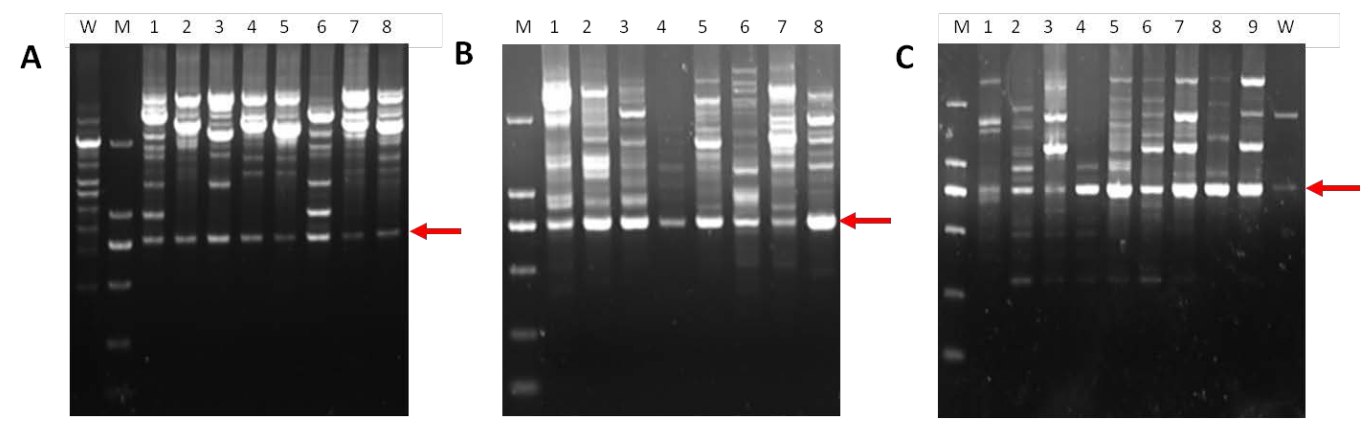

Figure 1. Random amplified polymorphic DNA (RAPD) amplification profile using primer. A. RAPD amplification of fresh samples. Lane M = DL 2000 marker; lanes 1-8 = J1-J8 of Gynostemma pentaphyllum; W: Cayratia japonica. Arrow: consistently amplified bands in G. pentaphyllum, J-750. B. RAPD amplification of dry samples. Lane $M=\mathrm{DL}$ 2000 marker; lanes 1-8 = JG1-JG8 of G. pentaphyllum. Arrow: consistently amplified bands from G. pentaphyllum, J-750. C. RAPD amplification of dry samples. Lane M = DL 2000 marker; lanes 1-9 = JG9-JG17 of G. pentaphyllum; W: C. japonica. Arrow: consistently amplified bands from G. pentaphyllum, J-750.

\section{Cloning and sequencing of RAPD marker}

To convert the specific RAPD marker to a SCAR marker for G. pentaphyllum, specific bands of DNA (about $750 \mathrm{bp}$ ) were amplified using primer 10 from eight fresh samples of $G$. pentaphyllum and were named J1-750-J8-750, respectively. Later, all fragments of J1-750-J8-750 were cloned in pMD18-T and sequenced. Restriction endonuclease (RE) digestion analysis revealed that a HindIII site existed in all J-750 samples, which could be used to cut the latter into two fragments of about 500 and $270 \mathrm{bp}$ on a $1.5 \% \mathrm{w} / \mathrm{v}$ agarose gel (Figure 2). The size of these 
amplified fragments was consistent with those of the corresponding RAPD. This means that the RAPD marker was successfully cloned. Subsequently, all sequences obtained of the RAPD marker were submitted to GenBank (accession No. HQ900672-HQ900679).

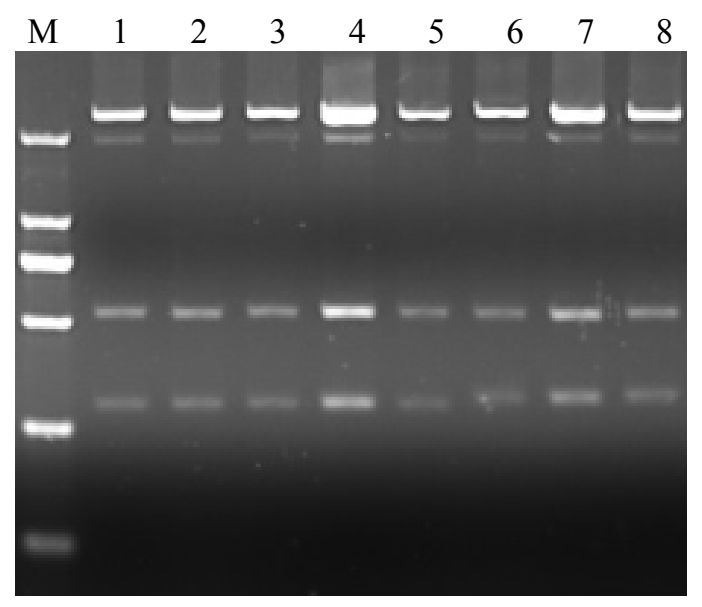

Figure 2. Double-restriction digestion profile from BamHI and Hindlll for eight recombinant plasmids of the J-750 random amplified polymorphic DNA (RAPD) amplicon. Lane $M=$ DL 2000 marker; lanes 1-8 = positive recombinant plasmids of J-750 from J1-J8 samples of Gynostemma pentaphyllum.

\section{Sequence analysis of RAPD amplicon}

The length and consistency of the eight RAPD amplicon sequences were analyzed (Table 4). These were highly conserved sequences with $>97 \%$ similarity. The length of the eight RAPD marker sequences ranged from 766 to $770 \mathrm{bp}$, comprising approximately $68 \% \mathrm{~A}-\mathrm{T}$. The results from BLAST analysis revealed that the homology of these sequences with known plant nucleotide sequences was at a low sequence-similarity level, $<40-90 \mathrm{bp}$. Two possible coding regions were found with 48 or 45 encoded amino acids. The function and possible protein-coding of two open reading frames requires further study. These eight sequences were found to contain several RE sites. The number of RE Sspl sites on J-750 were polymorphic, such that one Sspl site was found on J1-750 and J6-750, two Sspl sites on J3-750, J4-750, J5-750, J7-750, and J8-750, and three Sspl sites were found on J2-750. Most other RE digestion sites were single (Table S1).

\begin{tabular}{|c|c|c|c|c|c|c|c|c|c|}
\hline Length (bp) & Specimen No. & $\mathrm{J} 1-750(\%)$ & J2 -750 (\%) & J3-750 (\%) & J4-750 (\%) & J5-750 (\%) & J6-750 (\%) & J7-750 (\%) & J8-750 (\%) \\
\hline 769 & $\mathrm{~J} 1-750$ & 100.0 & & & & & & & \\
\hline 766 & $\mathrm{~J} 2-750$ & 97.7 & 100.0 & & & & & & \\
\hline 768 & J3-750 & 99.3 & 98.0 & 100.0 & & & & & \\
\hline 767 & J4-750 & 98.6 & 98.2 & 99.0 & 100.0 & & & & \\
\hline 770 & J5-750 & 98.3 & 97.4 & 98.4 & 98.8 & 100.0 & & & \\
\hline 769 & J6-750 & 99.9 & 97.8 & 99.5 & 98.7 & 98.4 & 100.0 & & \\
\hline 766 & J7-750 & 98.3 & 97.8 & 98.7 & 99.2 & 98.6 & 98.4 & 100.0 & \\
\hline 769 & J8-750 & 98.1 & 97.5 & 98.4 & 99.0 & 98.3 & 98.2 & 98.8 & 100.0 \\
\hline
\end{tabular}




\section{Amplification using SCAR primers}

Based on the eight J-750 RAPD marker sequences, SCAR primers were designed to amplify genomic DNA from 27 specimens (including two samples of $C$. japonica). Two pairs of control primers were used to amplify $18 \mathrm{~S}$ rDNA, and the length of the 18S rDNA fragment from G. pentaphyllum was $293 \mathrm{bp}$, and that from C. japonica was $177 \mathrm{bp}$. As shown in Figure 3A-C, a SCAR primer pair, G1 and G2, generated a single, distinct, and brightly resolved 359bp band in all G. pentaphyllum cultivars and dry materials, but no specific amplicons were observed from $C$. japonica species. Even though some degraded fragments were observed in the genomic DNA extracted from dry material, including commercial samples, SCAR bands were still strong and distinct for the specimens inspected. An internal control of approximately 290 bp for all G. pentaphyllum species and another of approximately $180 \mathrm{bp}$ for C. japonica species were observed on agarose gel (Figure $3 \mathrm{~A}-\mathrm{C}$ ). Therefore, these data show that it is possible to discriminate G. pentaphyllum from $C$. japonica using PCR amplification of SCAR and control primers (Table S2).

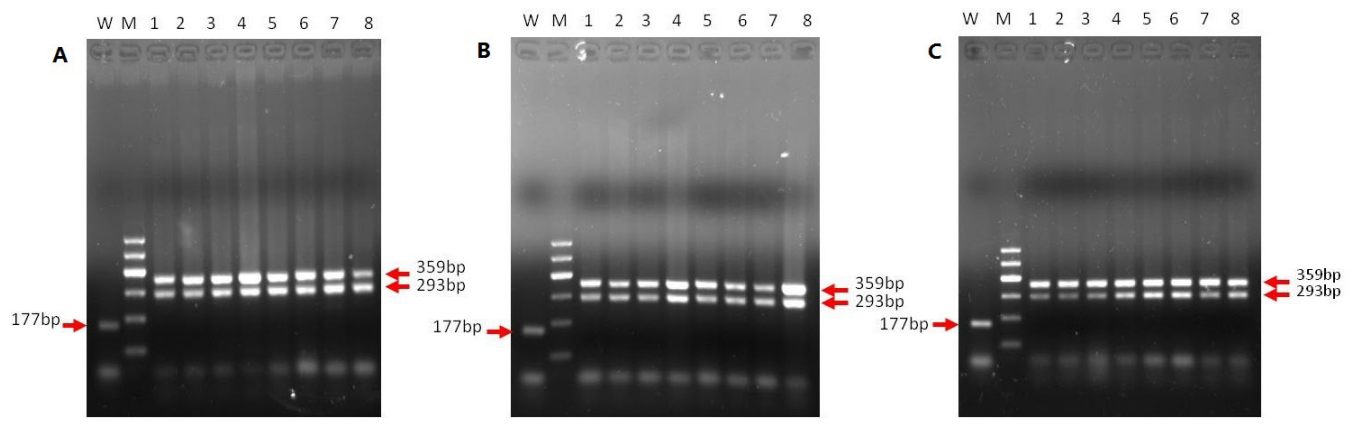

Figure 3. Amplification profile of sequence-characterized amplified region (SCAR) marker and control. A. Amplification of fresh samples. W: Cayratia japonica. Lane $M=$ marker 1; lanes 1-8 = J1-J8 samples from Gynostemma pentaphyllum. B. Amplification of dry samples. W: C. japonica; lane $M=$ marker 1; lanes 1-8 = JG1-JG8 dry samples of G. pentaphyllum. C. Amplification of dry samples. W: C. japonica; lane $M=$ marker 1; 1-8: JG10-JG18 dry samples of $G$. pentaphyllum.

\section{DISCUSSION}

Traditionally, common methods used to authenticate herbal medicines have depended on histological and morphological evaluations, but the accuracy of these methods is often influenced by environmental and subjective factors (Wang et al., 2012). Chemical analyses such as highperformance liquid chromatography and capillary electrophoresis are useful for identification; however, quantitative variations in characteristic compounds within herbal materials impede the confirmation of their botanical identities (Hon et al., 2003; Xie et al., 2011). It is clear that compounds within herbal medicines present chemical complexity, which are usually affected by growth, drying, and storage conditions, as well as the time of collection. Therefore, techniques involving molecular markers are more dependable as they are dependent on genetic composition and are not influenced by other factors (Wang et al., 2011b; Wu et al., 2011). 
RAPD analysis could be used to explore the high degree of genetic polymorphisms in various herbal medicines; not only is there no requirement for prior DNA sequence information of these species, but this technique is also easy to perform. In the present RAPD analysis, significant genetic polymorphism was observed among different specimens of G. pentaphyllum. The 19 random decamer primers screened could be used in research on germplasm resources. The genetic polymorphism in tested cultivars was distinguishable and could be utilized to develop markers for subsequent species identification. In this study, one consistent band of J-750 was amplified by random decamer primer 10 from $G$. pentaphyllum specimens but not from C. japonica. Therefore, this band could be selected as a genetic marker of G. pentaphyllum.

Based on sequence analysis of the cloned J-750 fragment, one pair of intraspeciesspecific primers (G1 and G2) specific for genomic DNA of G. pentaphyllum was designed and a SCAR marker amplification system was developed for the simultaneous authentication of $G$. pentaphyllum and $C$. japonica. The annealing site of $\mathrm{G} 1$ and $\mathrm{G} 2$ with the RAPD amplicon is shown in Figure 4. The size of the RAPD amplicon was sufficient for the layout of SCAR primers; meanwhile, the variable region could be evaded. Previously, we identified another species-specific RAPD amplicon (about 500 bp), but the length was not adaptive to conversion to a SCAR marker (data not shown). Here, J-750 was a more appropriate RAPD amplicon to be converted to a SCAR marker. In comparison with other assay methods of authentication, the detection of SCAR marker needs only a gel-based assay and requires no sequence analysis of the PCR products (Yang et al., 2013). In addition to SCAR marker primers, two pairs of control primers were used to amplify fragments of $18 \mathrm{~S}$ rDNA from G. pentaphyllum (J1 and J2) or C. japonica (W1 and W2), which were used as internal controls, respectively. The length of the SCAR marker is $359 \mathrm{bp}$, while the length of the control fragment from G. pentaphyllum and C. japonica was 293 and or $177 \mathrm{bp}$, respectively, and easily distinguished. In this assay, primer pairs G1-G2 and J1-J2 amplified the predicted 359- and 293-bp DNA fragments in all samples of G. pentaphyllum but not C. japonica. However, W1-W2 generated only a 177-bp fragment in C. japonica samples. Therefore, the SCAR marker amplification system is simple, reliable, repeatable, and cost-effective. The results substantiate the applicability of the SCAR amplification system as a qualitative diagnostic tool for the authentication and identification of $G$. pentaphyllum. Therefore, this system could be employed to discriminate crude and processed commercial materials of $G$. pentaphyllum from their adulterants.

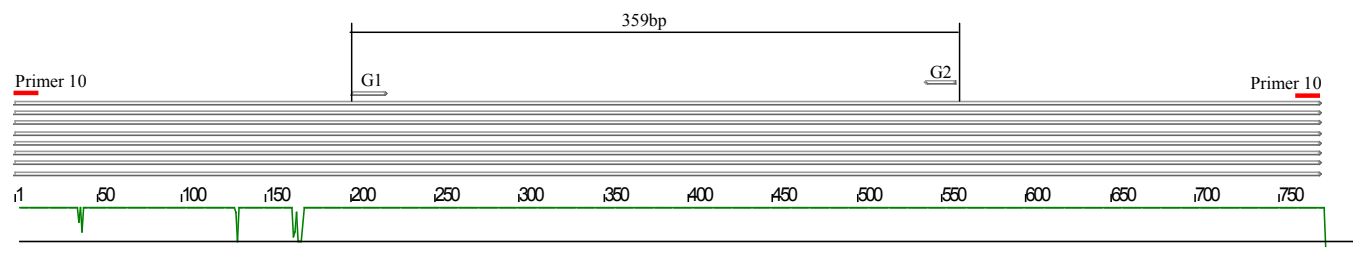

Figure 4. Alignment of species-specific primers G1 and G2 with random amplification polymorphic DNA (RAPD) marker amplicon.

Sequence analysis of the J-750 RAPD amplicon has revealed the presence of several restriction endonuclease sites. One of the RE sites, Sspl, is polymorphic in the inspected specimens of $G$. pentaphyllum, which could be developed as cleaved amplified polymorphic sequence and may be appropriate for the detection of single-nucleotide variations (Kim et al., 2006). In conclusion, the SCAR marker obtained in this study could be used for the identification of G. pentaphyllum, as 
well as for the simultaneous discrimination of $G$. pentaphyllum from $C$. japonica through the primer combination of two pairs of SCAR markers (G1-G2) and a control (J1-J2). Furthermore, the SCAR primers and the marker amplification system have been awarded the title of national patent by the Chinese State Bureau of Intellectual Property Office (ZL201110139269.4).

\section{Conflicts of interest}

The authors declare no conflict of interest.

\section{ACKNOWLEDGMENTS}

We are grateful to Prof. Kaijia He from Guangxi Institute of Chinese Medicine, and Researcher Zhengzhu Ling from Guangxi Medicine Botanical Garden for their help with sample identification of Gynostemma pentaphyllum and Cayratia japonica. Research supported in part by the Guangxi Natural Science Foundation (\#2010GXNSFA013145); the Scientific Research Project Foundation for High Education School from Guangxi Provincial Department of Education (\#201203YB038); and the National Natural Science Foundation of China (\#31260069) to Y.S. Wu.

\section{Supplementary material}

\section{REFERENCES}

Bashir KM, Awan FS, Khan IA, Khan Al, et al. (2014). Identification and authentication of Rosa species through development of species-specific SCAR marker(s). Genet. Mol. Res. 13: 4130-4139.

Chen ZD, Ren H and Wen J (2007). Flora of China (Vitaceae). Available at [http://www.efloras.org/florataxon.aspx?flora id=2\&taxon_id=10946].

Choi HS, Park MS, Kim SH, Hwang BY, et al. (2010). Neuroprotective effects of herbal ethanol extracts from Gynostemma pentaphyllum in the 6-hydroxydopamine-lesioned rat model of Parkinson's disease. Molecules 15: 2814-2824.

Deng $Q$ and Yang X (2014). Protective effects of Gynostemma pentaphyllum polysaccharides on PC12 cells impaired by MPP(+). Int. J. Biol. Macromol. 69: 171-175.

Ding BZ and Wang SY (1998). Flora of Henanica [M], Zhengzhou: Henan Science and Technology Publishing House.

Gao D, Zhao M, Qi X, Liu Y, et al. (2014). Hypoglycemic effect of Gynostemma pentaphyllum saponins by enhancing the Nrf2 signaling pathway in STZ-inducing diabetic rats. Arch. Pharm. Res. [Epub Ahead of Print].

Hon CC, Chow YC, Zeng FY and Leung FC (2003). Genetic authentication of ginseng and other traditional Chinese medicine. Acta Pharmacol. Sin. 24: 841-846.

Im SA, Choi HS, Choi SO, Kim KH, et al. (2012). Restoration of electric footshock-induced immunosuppression in mice by Gynostemma pentaphyllum components. Molecules 17: 7695-7708.

Jiang JF, Li XY, Wu YS, Luo Y, et al. (2009a). Fingerprints identification of Gynostemma pentaphyllum by RAPD and cloning and analysis of its specific DNA fragment. Zhong Yao Cai. 32: 190-193.

Jiang LY, Qian ZQ, Guo ZG, Wang C, et al. (2009b). Polyploid origins in Gynostemma pentaphyllum (Cucurbitaceae) inferred from multiple gene sequences. Mol. Phylogenet. Evol. 52: 183-191.

Kim DS, Kim DH, Yoo JH and Kim BD (2006). Cleaved amplified polymorphic sequence and amplified fragment length polymorphism markers linked to the fertility restorer gene in chili pepper (Capsicum annuum L.). Mol. Cells 21: 135-140.

Kim JK, An GH, Ahn SH, Moon YH, et al. (2012). Development of SCAR marker for simultaneous identification of Miscanthus sacchariflorus, M. sinensis and M. x giganteus. Bioprocess Biosyst. Eng. 35: 55-59.

Liao H, Zhao Y, Zhou Y, Wang Y, et al. (2011). Microsatellite markers in the traditional Chinese medicinal herb Gynostemma pentaphyllum (Cucurbitaceae). Am. J. Bot. 98: e61-e63.

Liu J, Zhang L, Ren Y, Gao Y, et al. (2014). Anticancer and immunoregulatory activity of Gynostemma pentaphyllum polysaccharides in $\mathrm{H} 22$ tumor-bearing mice. Int. J. Biol. Macromol. 69: 1-4.

Lu AM, Huang LQ, Chen SK and Charles J (2011). Flora of China (Cucurbitaceae). Available at [http://www.efloras.org/ florataxon.aspx?flora_id=2\&taxon_id=10233]. 
Mei ZQ, Fu SY, Yu HQ, Yang LQ, et al. (2014). Genetic characterization and authentication of Dimocarpus longan Lour. using an improved RAPD technique. Genet. Mol. Res. 13: 1447-1455.

Mizukami H, Okabe Y, Kohda H and Hiraoka N (2000). Identification of the crude drug atractylodes rhizome (Byaku-jutsu) and atractylodes lancea rhizome (So-jutsu) using chloroplast TrnK sequence as a molecular marker. Biol. Pharm. Bull. 23: 589-594.

Park SH, Huh TL, Kim SY, Oh MR, et al. (2014). Antiobesity effect of Gynostemma pentaphyllum extract (actiponin): a randomized, double-blind, placebo-controlled trial. Obesity 22: 63-71.

Ryuk JA, Kim YS, Lee HW and Ko BS (2014). Identification of Acorus gramineus, A. calamus, and A. tatarinowii using sequence characterized amplified regions (SCAR) primers for monitoring of Acori graminei rhizoma in Korean markets. Int. J. Clin. Exp. Med. 7: 2488-2496.

Schild L, Roth A, Keilhoff G, Gardemann A, et al. (2009). Protection of hippocampal slices against hypoxia/hypoglycemia injury by a Gynostemma pentaphyllum extract. Phytomedicine 16: 734-743.

Shan LN and Shi YX (2014). Effects of polysaccharides from Gynostemma pentaphyllum (Thunb.), Makino on physical fatigue. Afr. J. Tradit. Complement. Altern. Med. 11: 112-117.

Sun Q, Yang X and Li R (2014). SCAR marker for sex identification of Pistacia chinensis Bunge (Anacardiaceae). Genet. Mol. Res. 13: 1395-1401.

Tsui KC, Chiang TH, Wang JS, Lin LJ, et al. (2014). Flavonoids from Gynostemma pentaphyllum exhibit differential induction of cell cycle arrest in H460 and A549 cancer cells. Molecules 19: 17663-17681.

Wang DW, Li Y and Li ZQ (2011a). Identification of a male-specific Amplified Fragment Length Polymorphism (AFLP) and a Sequence Characterized Amplified Region (SCAR) marker in Eucommia ulmoides Oliv. Int. J. Mol. Sci. $12: 857-864$.

Wang H, Kim MK, Kwon WS, Jin H, et al. (2011b). Molecular authentication of Panax ginseng and ginseng products using robust SNP markers in ribosomal external transcribed spacer region. J. Pharm. Biomed. Anal. 55: 972-976.

Wang H, Kim MK, Kim YJ, Lee HN, et al. (2012). Molecular authentication of the Oriental medicines Pericarpium citri reticulatae and Citri unshius pericarpium using SNP markers. Gene 494: 92-95.

Wang M, Wang F, Wang Y, Ma X, et al. (2013). Metabonomics study of the therapeutic mechanism of Gynostemma pentaphyllum and atorvastatin for hyperlipidemia in rats. PLoS One 8: e78731.

Wu PK, Tai WC, Choi RC, Tsim KW, et al. (2011). Chemical and DNA authentication of taste variants of Gynostemma pentaphyllum herbal tea. Food Chem. 128: 70-80.

Xie Z, Zhao Y, Chen P, Jing P, et al. (2011). Chromatographic fingerprint analysis and rutin and quercetin compositions in the leaf and whole-plant samples of di- and tetraploid Gynostemma pentaphyllum. J. Agric. Food Chem. 59: 3042-3049.

Xin GZ, Lam YC, Maiwulanjiang M, Chan GK, et al. (2014). Authentication of Bulbus Fritillariae Cirrhosae by RAPD-derived DNA markers. Molecules 19: 3450-3459.

Yang L, Fu S, Khan MA, Zeng W, et al. (2013). Molecular cloning and development of RAPD-SCAR markers for Dimocarpus longan variety authentication. Springerplus 2: 501. 